\title{
Pressure Calibration to 100 kbar Based on the Compression of $\mathrm{NaCl}$
}

\author{
R. N. Jeffrey \\ J. Dean Barnett \\ jdeanbarnett@gmail.com \\ H. B. Vanfleet \\ H. Tracy Hall
}

Follow this and additional works at: https://scholarsarchive.byu.edu/facpub

Part of the Astrophysics and Astronomy Commons, and the Physics Commons

\section{Original Publication Citation}

Jeffery, R. N., J. D. Barnett, H. B. Vanfleet, and H. T. Hall."Pressure Calibration to 1 kbar Based on the Compression of NaCl." Journal of Applied Physics 37 (1966): 3172-318.

\section{BYU ScholarsArchive Citation}

Jeffrey, R. N.; Barnett, J. Dean; Vanfleet, H. B.; and Hall, H. Tracy, "Pressure Calibration to 100 kbar Based on the Compression of $\mathrm{NaCl}$ (1966). Faculty Publications. 807.

https://scholarsarchive.byu.edu/facpub/807

This Peer-Reviewed Article is brought to you for free and open access by BYU ScholarsArchive. It has been accepted for inclusion in Faculty Publications by an authorized administrator of BYU ScholarsArchive. For more information, please contact ellen_amatangelo@byu.edu. 


\section{Tungsten}

The work function data for two 3-mil $99.995 \%$ pure ribbons measured at 22 temperatures between $1150^{\circ}$ and $2200^{\circ} \mathrm{K}$ are listed in Table I. Both ribbons were outgassed at $2400^{\circ} \mathrm{K}$, one for $15 \mathrm{~h}$ and one for $18 \mathrm{~h}$. The work function was $4.52( \pm 0.07) \mathrm{eV}$ for both surfaces which were $96 \%$ and $95 \%$ (100)-oriented, with the remainder almost entirely (111).

\section{Rhenium}

The work function of a 2 -mil $99.99+\%$ pure Re ribbon outgassed at $2225^{\circ} \mathrm{K}$ for $10 \mathrm{~h}$ and measured at 10 temperatures between $1325^{\circ}$ and $2250^{\circ} \mathrm{K}$ was $4.96( \pm 0.05) \mathrm{eV}$. The crystallography of the rhenium ribbon following all emission measurements was fairly random, with all of the many hcp faces present, but with enhancement of the (001), (212), and the (101), (102), (103), (104), (105), $\cdots$ series.

\section{Osmium}

An osmium emitter was fabricated by hot pressing and sintering a disk to $95 \%$ of theoretical density from high-purity Os powder. The disk was cut, polished, and bored into a square-cross-section emitter with a central hole, through which was placed a high-purity beryllia insulator to house a tungsten heater. Osmium cannot be rolled into a foil or ribbon. The Os was outgassed at $1570^{\circ} \mathrm{K}$ for $3.8 \mathrm{~h}$, followed by $2.3 \mathrm{~h}$ at $1610^{\circ} \mathrm{K}$, followed by $1.4 \mathrm{~h}$ at $1680^{\circ} \mathrm{K}$. The processing at the highest temperature was continued until the emission stabilized; it decreased during the processing. The work function was measured at 11 temperatures in both increasing and decreasing temperature sequences between $1400^{\circ}$ and $1650^{\circ} \mathrm{K}$. The results, taken from the cup collector only, were $4.83( \pm 0.05) \mathrm{eV}$ (see Table I), with a distinct negative temperature coefficient expressed by $\phi=5.93-3.9 \times 10^{-4} \mathrm{~T} \mathrm{eV}( \pm 0.03 \mathrm{eV}) . \mathrm{X}$-ray diffraction measurements performed prior to and following the emission measurements indicated identical random hcp orientation.

\section{Iridium}

The work function of a 2-mil $99.9 \%$ pure Ir ribbon was measured at 13 temperatures between $1300^{\circ}$ and $2100^{\circ} \mathrm{K}$, following outgassing at $2100^{\circ} \mathrm{K}$ for $9 \mathrm{~h}$. The results, shown in Table $\mathrm{I}$, were $5.27( \pm 0.05) \mathrm{eV}$ for the fcc surface which was about $80 \%$ (111)-oriented with most of the remainder (100).

It is noted that of the four bcc metals, three were oriented by thermal treatment to $(100)$, and the fourth (Ta) was (211). The only fcc metal studied (Ir) became (111)-oriented. The two hcp metals remained largely random, although the Os was not heated hot enough to cause thermal preferential orientation.

\title{
Pressure Calibration to $100 \mathrm{kbar}$ Based on the Compression of $\mathrm{NaCl}^{*}$
}

\author{
R. N. Jefrerey, $\dagger$ J. D. Barnett, H. B. Vanfleet, and H. T. Hall \\ Departments of Physics and Chemistry, Brigham Young University, Provo, Utah
}

(Received 21 February 1966)

\begin{abstract}
An independent determination has been made of the transformation pressures assigned to the highpressure transformations in $\mathrm{Ba}$ and $\mathrm{Bi}$ which are generally used for calibration points. The metals were imbedded in $\mathrm{NaCl}$, and the transitions were detected by the resistance change of the metal while the $\mathrm{NaCl}$ lattice parameter was simultaneously measured by $\mathrm{x}$-ray diffraction. The $\mathrm{NaCl}$ compression values determined at the fixed points were related to pressure by reference to the semiempirical compression curve of Decker. The $\mathrm{Ba} \mathrm{I}-\mathrm{II}$ and $\mathrm{Bi}$ III-V transformations at room temperature were assigned thermodynamic equilibrium values of $53.3 \pm 1.2 \mathrm{kbar}$ and $73.8 \pm 1.3 \mathrm{kbar}$, respectively, corresponding to $\mathrm{NaCl}$ linear compression values of $\Delta a / a_{0}=0.0510$ and 0.0637 , respectively. Hysteresis in the transformation pressures was investigated and sample hysteresis was separated from apparatus hysteresis. The hysteresis measurements imply a stress energy which inhibits nucleation of the new phase and causes a sizable sample hysteresis in solid-media systems.
\end{abstract}

\section{INTRODUCTION}

A CCURATE calibration of very high pressure devices has been a major problem since the early days of high-pressure research. In simple piston-

* Supported by the U.S. Army Research Office, Durham, North Carolina.

$\dagger$ Present address: Physics Department, University of Illinois, Urbạna, Illinois, cylinder devices, pressure can be calculated from applied force per unit piston area if sufficient corrections are included. In many high-pressure devices, particularly the solid-media systems, such a direct calculation of pressure is not possible, and a calibration must be made by other means. The pressure calibration generally used is based upon the observation of certain "sharp" phase transitions which occur in materials such as $\mathrm{Tl}, \mathrm{Ba}$, and 
Bi. ${ }^{1}$ The pressures at which these transitions occur is taken from previous measurements in an apparatus where pressures could be determined. Although no precise, fundamental determination of the points above $50 \mathrm{kbar}$ has been carried out, the fixed-point calibration technique is in widespread use.

Bridgman, in volume displacement ${ }^{2}$ and electrical resistance $^{3}$ measurements, determined pressures by direct calculation based on force per unit area in two different pressure systems. Although he never intended these measurements to represent precision calibration values, his observations of phase transitions were used to designate two different pressure scales, the so-called "volume" and "resistance" scales. Bridgman had forced agreement between the two scales below $30 \mathrm{kbar}$, but rather large discrepancies existed at higher pressures. For example, a volume discontinuity was reported for $\mathrm{Ba}$ at $59 \mathrm{kbar}$, and a resistance discontinuity in $\mathrm{Ba}$ was reported at $78 \mathrm{kbar}$. Kennedy and LaMori ${ }^{4}$ first proposed these two transitions to be the same phase transformation and the correct pressure values to be given by the volume scale. Barnett, Bennion, and Hall ${ }^{5}$ demonstrated experimentally the identity of these two transitions by measuring the volume and resistance of Ba simultaneously, although no pressure calibration was attempted. Kennedy and LaMori ${ }^{6}$ later redetermined the transition pressures of $\mathrm{Bi}, \mathrm{Tl}$, and $\mathrm{Cs}$ with pressure to 50 kbar. Because of the generally good agreement with Bridgman's volume scale, the latter is currently accepted as the calibration standard with minor corrections from Kennedy and LaMori's work. Errors in the resistance scale are now felt to be due to the unknown pressure distribution in the Bridgman anvil device used in the resistance measurements. Attempts to check the Ba point at $59 \mathrm{kbar}$ by an independent method have not been conclusive, and no attempts at independent pressure determinations in static pressure systems have been made at pressures above 60 kbar. Recent work by Stark and Jura, ${ }^{7}$ Giardini and Samara, ${ }^{8}$ and others indicates serious discrepancies between Bridgman's pressure value of the upper $\mathrm{Bi}$ transition and calibration curves based on the Ba point.

${ }^{1}$ F. P. Bundy, in Modern Very High Pressure Techniques, R. H. Wentorf, Jr., Ed. (Butterworth Scientific Publications, Washington, D. C. 1962) pp. 18-21.

${ }^{2}$ P. W. Bridgman, Proc. Am. Acad. Arts Sci. 74, 425 (1942), and Phys. Rev. 60, 351 (1941).

${ }^{3}$ P. W. Bridgman, Proc. Am. Acad. Arts Sci. 81, 167 (1952).

4 G. C. Kennedy and P. N. LaMori, in Progress in Very High Pressure Research, F. Bundy, W. Hibbard, and H. Strong, Eds.

(John Wiley \& Sons, New York, 1961), pp. 304-314.

${ }^{5}$ J. D. Barnett, R. B. Bennion, and H. 'T. Hall, Science 141, 534 (1963).

${ }^{6}$ G. C. Kennedy and P. N. LaMori, J. Geophys. Res. 67, 851 (1962).

${ }^{7}$ W. Stark and G. Jura, ASME paper $64-\mathrm{Wa} / \mathrm{Pt}-28$, presented at winter meeting 1-3 December 1964, New York.

${ }^{8}$ A. A. Giardini and G. A. Samara, J. Phys. Chem. Solids 26, 1523 (1965).
Drickamer and co-workers ${ }^{9,10}$ have reported static pressures of several hundred kilobars using supported Bridgman anvils for resistance and $\mathrm{x}$-ray studies. The resistance cell ${ }^{9}$ was calibrated by extrapolating Bridgman's resistance data on $\mathrm{Pb}, \mathrm{Pt}$, and In and using the $\mathrm{Ba}$ and high $\mathrm{Bi}$ transitions (volume scale) as fixed points. The x-ray cell10 was calibrated by observing the change in lattice parameter of a "marker" of $\mathrm{Ag}, \mathrm{Rh}$, or Pd and comparing with compression data obtained in shock measurements. By shock techniques ${ }^{11}$ dynamic pressures of over a megabar have been obtained where pressures are calculated using the basic conservation laws of mechanics and measuring shock velocities. Apparent support for the accuracy of the Drickamer resistance-cell calibration was found in the observation of a transition in $\mathrm{Fe}$ at $133 \mathrm{kbar}$, corresponding to a shock-induced transformation measured at 131 kbar. $^{12}$ This apparent agreement is in contradiction to the results obtained in the present work as described below and again raises the fundamental question of equality of static equilibrium, quasihydrostatic pressures in the solid systems, and the unidirectional pressures created in the dynamic shock front. Evidence is accumulating to indicate that the pressure in shock measurements has a much higher shear component than is present in the static systems, and especially in those static systems with a three-dimensional pressure chamber. The amount of shear in the Bridgman anvil device is still undetermined but is undoubtedly much greater than in the three-dimensional high-pressure systems in use.

Due to the inherent pressure limitations of pistoncylinder devices, it is commonly felt that for pressures above 50 kbar a calibration must be obtained by some other technique. We propose the use of a pressure scale based on the atomic separation of a simple substance for which the compression can be theoretically predicted with some degree of certainty. $\mathrm{NaCl}$ was chosen for this substance because it is theoretically amenable, experimentally easy to work with, and there is a large amount of accurate data available to support the theory. One advantage of such an approach is that the pressure calibration points are tied to a physical parameter independent of any apparatus and, in fact, independent of any theory. We report herein the actual compression values of $\mathrm{NaCl}$. We then relate these values to the absolute pressure scale by reference to the semiempirical theoretical equation of state for $\mathrm{NaCl}$ calculated by Decker. ${ }^{13}$ Should an improved theoretical treatment become available, the measured compression values would yield an improved absolute pressure scale.

${ }^{9}$ A. S. Balchan and H. G. Drickamer, Rev. Sci. Instr. 32, 308 (1961).

${ }^{10}$ E. A. Perez-Albuerne, K. F. Forsgren, and H. G. Drickamer, Rev. Sci. Instr. 35, 29 (1964).

1 G. E. Duvall and G. R. Fowles, High Pressure Physics and Chemistry (Academic Press Inc., New York, 1963), Vol. II, pp. 209-291.

12 D. Bancroft, E. L. Peterson, and S. Minshall, J. Appl. Phys. 27, 291 (1956).

is D. L. Decker, J. Appl. Phys. 36, 157 (1965). 

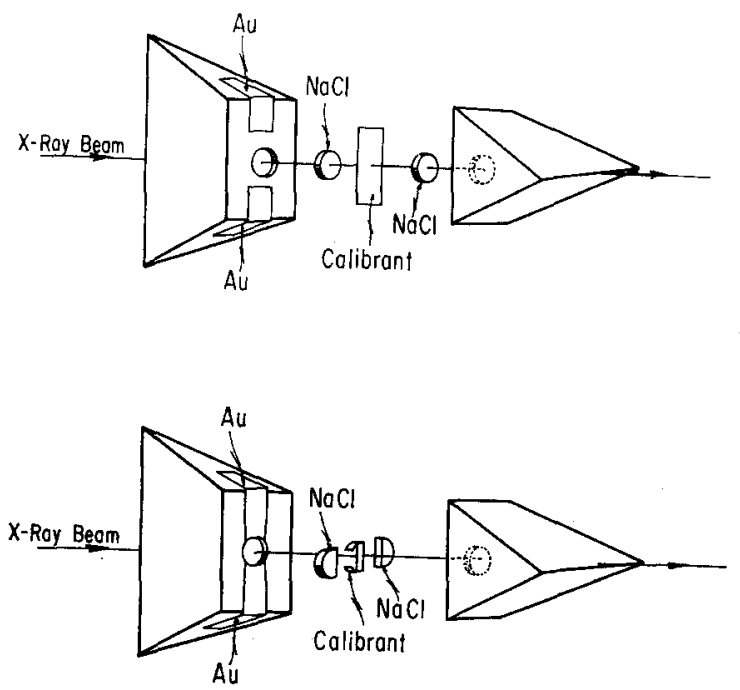

Frg. 1. Construction of calibration samples showing location of calibrant, $\mathrm{NaCl}$, and x-ray beam.

Decker's equation of state has been solved to yield pressure as a function of lattice parameter and temperature over the range $0-500 \mathrm{kbar}$ and $0^{\circ}-1500^{\circ} \mathrm{C}$. Lattice parameters have been measured at high pressure using the $x$-ray diffraction tetrahedral press described previously. ${ }^{14}$

The use of a lattice parameter as a pressure gauge as discussed herein has several major advantages over the fixed-point technique for use in high-pressure studies. First, it is a single-valued variable with pressure; hence, the pressure may be known for both increasing and decreasing pressure cycles of a particular experiment or after a number of cycles. This makes possible a direct observation of hysteresis effects in the apparatus, and it allows one to separate, for example, the intrinsic sample hysteresis in phase transformations from apparatus hysteresis. Second, the lattice parameter is a continuous variable with pressure, which allows one to obtain pressure values over an extended range with one calibrant and to measure pressures at any desired working pressure of interest. Third, the calibrant is located within the pressure chamber in intimate contact with the sample. One can thus observe local pressure changes due to effects within the pressure chamber itself, independent of oil pressure. These advantages, shared with the manganin gauge and similar devices, have been taken for granted by workers using hydrostatic liquid systems, but the lack of them has been a serious limitation in high-pressure studies with solidmedia environments.

The primary purpose of this paper is to determine the calibration values of the fixed-point calibration transitions, in particular the $\mathrm{Ba}$ I-II and the Bi III-V transitions. It has been necessary, however, to discuss hysteresis effects in these transformations since they

\footnotetext{
${ }^{14}$ J. D. Barnett and H. T. Hall, Rev. Sci. Instr. 35, 175 (1964).
}

have a bearing on the interpretation of the results. In addition, the results of this work have value as an important link between theory and experiment for $\mathrm{NaCl}$ when correlation of other pressure-calibration techniques at the lower pressures are taken into account.

Much of the inherent advantage of the techniques described herein would be lost if $\mathrm{NaCl}$ were itself to exhibit a phase transformation under high pressure. The transformation previously reported by Evdokimova and Vereshchagin ${ }^{15}$ has not been observed in our laboratory using the tetrahedral apparatus, even to pressures of $100 \mathrm{kbar}$ at $25^{\circ} \mathrm{C}$ for periods up to a week, and at $30 \mathrm{kbar}$ at $600^{\circ} \mathrm{C}$ for several hours. Other workers in the field have also searched in vain for this transition..$^{16}$ It seems probable that if such a transition does exist, it is initiated by shearing forces present in the apparatus. A recent indication of this transformation by shock measurements ${ }^{17}$ seems to indicate that this is the case. Since our measurements are based on the x-ray diffraction lines of the low-pressure form, and since we see no indication of the transformation in our apparatus on a microscopic level, we have full confidence in the data reported here.

\section{TECHNIQUES}

Several inherent features of the tetrahedral $x$-ray diffraction apparatus make it feasible to evaluate the fixed transition pressures in the manner described herein. First, the pressure resolution is greatly superior to other pressure devices. This resolution is clearly demonstrated in the work on cesium metal by Hall, Merrill, and Barnet ${ }^{18}$ wherein the cusp in the resistancevs-pressure curve of Bridgman near $42 \mathrm{kbar}$ was actually found to be a flat-topped spike only $0.5 \mathrm{kbar}$ wide. This measurement implies that pressure gradients over a sizable volume are much less than $0.5 \mathrm{kbar}$, a condition highly desirable in a calibration study. Second, the precision of lattice parameter determinations made using the " $\mathrm{B}$ " $\mathrm{x}$-ray geometry of the press ${ }^{14}$ is approximately $0.1 \%$. When used in connection with the $\mathrm{NaCl}$ compression curve, this represents a pressure difference of less than $1.0 \mathrm{kbar}$. Third, simultaneous $\mathrm{x}$-ray and electrical resistance measurements can be made, thus allowing the measurement of the $\mathrm{NaCl}$ compression coincident with the observation of the resistance transitions in the calibration materials in the same manner as generally used.

The metal calibration specimen was imbedded in $\mathrm{NaCl}$ to insure equal pressure in both materials and was placed in such a position that the x-ray beam passed through the $\mathrm{NaCl}$ which was in intimate contact with

${ }^{15}$ V. V. Evdokimova and L. F. Vereshchagin, Soviet Phys.-JETP 16, 855 (1963).

${ }^{16} \mathrm{~J}$. C. Jamieson, in Physics of Solids at High Pressure, C. T. Tomizuka, and R. M. Emrick, Eds. (Academic Press Inc., New York, 1965), pp: 444-459.

${ }^{17}$ D. B. Larson et al., J. Phys. Chem. Solids 27, $476(1966)$.

${ }_{18}$ H. T. Hall, L. Merrill, and J. D. Barnett, Science 146, 1297 (1964). 
TABLE I. NaCl linear compressions and corresponding pressures from Decker's theory with sample hysteresis and a comparison of previously accepted values for each transition.

\begin{tabular}{|c|c|c|c|c|c|c|c|c|c|}
\hline \multirow[b]{2}{*}{ Transition } & \multicolumn{3}{|c|}{$\% \mathrm{NaCl}$ compression at transition } & \multicolumn{4}{|c|}{ Corresponding pressure (kbar) } & \multirow[b]{2}{*}{$\begin{array}{l}\text { Bridgman's } \\
\text { volume }^{\mathrm{a}}\end{array}$} & \multirow[b]{2}{*}{$\begin{array}{l}\text { Discrepancy } \\
\left(P_{B}-P_{\mathrm{NaCl}}\right)\end{array}$} \\
\hline & $\begin{array}{l}\text { Increas- } \\
\text { ing }\end{array}$ & $\begin{array}{l}\text { Decreas- } \\
\text { ing }\end{array}$ & $\begin{array}{l}\text { Equi- } \\
\text { librium }\end{array}$ & $\begin{array}{c}\text { Increas- } \\
\text { ing }\end{array}$ & $\begin{array}{l}\text { Decreas- } \\
\text { ing }\end{array}$ & $\begin{array}{c}\text { Hys- } \\
\text { teresis }\end{array}$ & $\underset{\text { librium }^{\mathbf{b}}}{\text { Equi- }}$ & & \\
\hline Bi I-II (Strip) & $2.97 \pm 0.07$ & $2.70 \pm 0.07$ & $2.84 \pm 0.07$ & $26.2 \pm 0.8$ & $23.4 \pm 0.8$ & 2.8 & $24.8 \pm 0.8$ & 25.4 & 0.6 \\
\hline Bi I-II (Wire) & $2.99 \pm 0.12$ & $2.49 \pm 0.12$ & $2.74 \pm 0.12$ & $26.5 \pm 1.3$ & $21.2 \pm 1.2$ & 5.3 & $23.8 \pm 1.3$ & 25.4 & 1.6 \\
\hline Bi II-III (Strip) & $3.23 \pm 0.07$ & $3.03 \pm 0.07$ & $3.13 \pm 0.07$ & $29.1 \pm 0.8$ & $26.9 \pm 0.8$ & 2.2 & $28.0 \pm 0.8$ & 27.0 & -1.0 \\
\hline Bi II-III (Wire) & $3.28 \pm 0.12$ & $2.89 \pm 0.12$ & $3.09 \pm 0.12$ & $29.7 \pm 1.4$ & $25.5 \pm 1.3$ & 4.2 & $27.6 \pm 1.4$ & 27.0 & -0.6 \\
\hline TI II-III & $3.77 \pm 0.17$ & $3.63 \pm 0.17$ & $3.70 \pm 0.17$ & $35.4 \pm 2.1$ & $33.8 \pm 2.1$ & 1.6 & $34.6 \pm 2.1$ & 36.7 & 2.1 \\
\hline $\mathrm{Yb}$ I-II & $3.99 \pm 0.12$ & $1.82 \pm 0.12$ & & $38.2 \pm 1.5$ & $14.7 \pm 1.1$ & $\cdots$ & $\ldots$ & $\cdots$ & $\cdots$ \\
\hline Ba I-II & $5.19 \pm 0.06$ & $5.01 \pm 0.10$ & $5.10 \pm 0.08$ & $54.6 \pm 0.9$ & $52.0 \pm 1.5$ & 2.6 & $53.3 \pm 1.2$ & 58.8 & 5.5 \\
\hline Bi III-V & $6.49 \pm 0.07$ & $6.27 \pm 0.07$ & $6.37 \pm 0.07$ & $75.7 \pm 1.3$ & $71.9 \pm 1.3$ & 3.8 & $73.8 \pm 1.3$ & 88 & 14.2 \\
\hline Sn I-II & $7.36 \pm 0.15$ & $\ldots$ & $\ldots$ & $92 \pm 3$ & 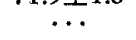 & $\cdots$ & 1. & 114 & 22 \\
\hline
\end{tabular}

a $\mathrm{Bi}$ and $\mathrm{Tl}$ taken from Ref. 6 and Sn from Ref. 25 in which correlation is made to Bridgman's volume scale.

b Average of incresing and decreasing transition pressure as "best" estimate of equilibrium value from data. See text for details on Bi.

the metal. The x-ray beam itself is approximately 0.020 in. wide. Two geometries used are illustrated in Fig. 1. The sample of Fig. 1 (top) allows for x-ray diffraction patterns to be recorded for both the $\mathrm{NaCl}$ and metal specimens simultaneously while the resistance of the metal is being measured. Volume and resistance transitions in the metal are thus measured coincidently. For the sample of Fig. 1 (bottom) only the $\mathrm{NaCl}$ diffraction pattern is recorded, and the transition in the metal is detected by the resistance change alone. The $\mathrm{Ba}$ transitions were recorded using both techniques whereas the $\mathrm{Bi}$ were recorded using only the latter. As the calibration points were determined, the pressure was increased very slowly as the transition point was approached.

All of the calibration experiments have been conducted using 50-50 wt\% boron-plastic tetrahedra described elsewhere. ${ }^{14}$ Two different anvil sizes were used depending upon maximum pressures desired. Three-quarter-inch anvils were used with 1-in. tetrahedra for most experiments in the range from 0-50 kbar. For experiments to $100 \mathrm{kbar}$, a modified anvil was used which was täpered in the normal gasket region such as to produce a wedge-shaped gasket of $8^{\circ}$ angle followed by the normal parallel gasket area. The face of the anvil was $\frac{5}{16}$ in. along the triangular edge, and the tapered area was $0.150 \mathrm{in}$. wide. It has been our experience that having the positive-wedge region before the parallel-gasket region results in greater gasket stability at higher pressures.

\section{MEASUREMENTS}

The major effort of this work was directed toward determining the pressures asssociated with the Ba I-II and the Bi III- $\mathrm{V}$ transformations since these two calibration points are the most widely used, and the higher pressure data are almost completely based upon them. One measurement each was also made on the $\mathrm{Tl}, \mathrm{Yb}$, and $\mathrm{Sn}$ transitions as supporting data. An associated study was also made of the Bi I-II and Bi II-III transitions to determine hysteresis effects and to check on the accuracy of Decker's theory. During many of our high-pressure experiments, $\mathrm{x}$-ray data were taken at regular intervals to determine the efficiency of pressure transmission of the tetrahedral press, the characteristics of the pressure release cycle, and pressure cycling effects. A compilation of the $\mathrm{NaCl}$ linear compression data taken at each of the seven transformations studied is given in Table $\mathrm{I}$. The values for $\mathrm{Ba}$ and for the three $\mathrm{Bi}$ transformations represent the average of several measurements taken on both increasing and decreasing pressure cycles as indicated. Only one measurement was made on each of the other three transformations. The actual $\mathrm{NaCl}$ linear compressions are given for future reference, as well as the associated hysteresis intervals and equilibrium pressure values as determined using Decker's theory. All measurements correspond to a temperature of $25^{\circ} \mathrm{C}$. The nomenclature for the $\mathrm{Bi}$ III-V transition reported at $88 \mathrm{kbar}$ by Bridgman in volume measurements follows that of Klement, Jayaraman, and Kennedy. ${ }^{19}$ This transition is commonly known as the "upper bismuth" transition and is clearly the "VI-VIII" transition reported by Bundy ${ }^{20}$ at 122.5 kbar on the resistance scale.

Due to the magnitude of the sample hysteresis effects measured in this work, an understanding of the hysteresis phenomena becomes important in relationship to calibration of high-pressure systems by the fixedpoint method since sizable errors can result even when one has a calibrant within the pressure chamber and measurements are being made near one of the calibration points. The error intervals associated with the $\mathrm{NaCl}$ lattice compressions in Table I arise from two sources: (a) the variation of lattice parameter as determined by various lines in the $\mathrm{x}$-ray pattern and (b) the variation of the determined parameter from experiment to experiment. For $\mathrm{Ba}$ and $\mathrm{Bi}$, different orientations and configurations were used to demonstrate any systematic errors, but these variations were of the same order as the experiment to experiment

${ }^{19}$ W. Klement, Jr., A. Jayaraman, and G. C. Kennedy, Phys. Rev. 131, 632 (1963).

${ }^{20}$ F. P. Bundy, Phys. Rev. 110, 314 (1958). 


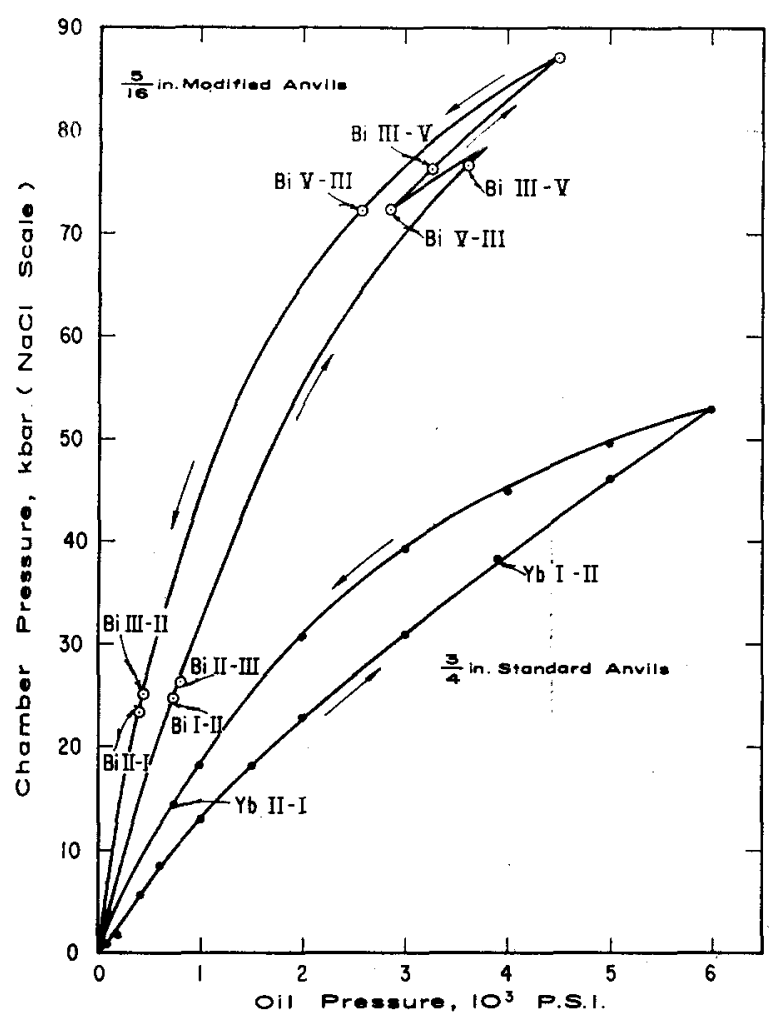

Frg. 2. Apparatus load vs pressure curves for the two different anvil sizes for both increasing and decreasing pressures. The separation of apparatus hysteresis from sample hysteresis in the $\mathrm{Yb}$ I-II and in the three Bi transitions is clearly evident.

variations and are thus included in (b) above. Since each diffraction line determines a lattice parameter with compound errors associated with both sources above, the error interval was evaluated by assuming the error in each lattice parameter obtained from each diffraction line of all patterns at a given transformation was Gaussian. An estimate was made of the standard deviation $\sigma$ for this statistical error by analyzing the Bi III-IV and Ba I-II data in which several independent experiments were made each involving several $\mathrm{x}$-ray lines. The error interval given in Table $\mathrm{I}$ is $2 \sigma_{n}$ where $\sigma_{n}=\sigma / \sqrt{ } n$ is the estimated standard deviation of the mean of $n$ measurements. Error intervals in pressure values were taken from corresponding errors in $\mathrm{NaCl}$ compressions using Decker's theory. The decrease in compressibility at higher pressures thus yields higher pressure error intervals at the higher pressure points. If absolute pressures are desired, an allowance reflecting the reliability of the theory must also be made in addition to the experimental errors given here.

\section{A. Apparatus Calibration}

A typical calibration curve for a solid-media highpressure apparatus gives sample pressure as a function of ram load or hydraulic oil pressure. Calibration curves for the tetrahedral apparatus with $\frac{3}{4}$-in. anvils and the modified $\frac{5}{16}$ in. anvils discussed above are shown in
Fig. 2. The lower and upper curves, corresponding to the $\frac{3}{4}$ in. and $\frac{5}{16}$ in. anvils, respectively, were obtained during calibration experiments on $\mathrm{Yb}$ and $\mathrm{Bi}$. The points at which the transitions occurred are indicated, both on the increasing and decreasing cycles, and the effect of pressure cycling around the upper $\mathrm{Bi}$ transition is also indicated. The anomalous curvature at very low pressures for the $\frac{3}{4}$ in. anvils and the decreasing efficiency at higher pressures, especially for the $\frac{5}{16}$ in. anvils, are clearly revealed. The loss of efficiency at higher pressures which has of ten been discussed qualitatively by various workers is in direct contrast to the linear calibration curves often assumed, and implies that at the higher pressures, previously reported values have been overestimated. Apparatus hysteresis due to gasket friction is clearly shown in Fig. 2 by the displacement of the calibration curve to the left on the decreasing pressure cycle. Sample hysteresis in the $\mathrm{Yb}$ and three Bi transformations is also illustrated on the graph by the vertical displacement of the transition point between the increasing and decreasing cycles. This clearly shows the separation of the apparatus hysteresis from sample hysteresis, and in the case of $\mathrm{Yb}$ defines the region of metastability. This same measurement was made on each of the transitions studied, and a measurable amount of sample hysteresis has been exhibited in each case, although none is as dramatic as Yb. Such measurements have not previously been possible in solid pressure systems. The curve for the $\frac{3}{4}$ in. anvils shown in Fig. 2 is reproducible in actual pressure values at a given load within $2 \mathrm{kbar}$ on the increasing pressure cycle, but the curve for the $\frac{5}{16}$ in. modified anvils is only representative, since pressures at a given load are not as consistent with the smaller anvils. Apparently the calibration curve depends rather critically on the initial size of the tetrahedron used, and adequate control has not been exercised in this regard. The use of a calibration curve without reference to an internal calibrant is not as meaningful for the small anvil design as with the standard size tetrahedron.

\section{B. Hysteresis Phenomena}

In the initial measurement on the Bi I-II transitions made to establish confidence in the technique and the theory, sizable sample hysteresis was observed even though the average of the up and down cycle pressure determinations agreed well with the accepted equilibrium value for the transformation. Further work indicated a larger amount of hysteresis for cylindrical wire samples than for flat strip samples of $\mathrm{Bi}$ when imbedded in $\mathrm{NaCl}$. The transitions are also "sharper" for strips than for wires when imbedded in $\mathrm{NaCl}$. Under liquid hydrostatic conditions, Bridgman ${ }^{21}$ measured a "region of indifference" of from 60 to 100 bars necessary to cause the transition to "run" in the two opposite directions starting from an equilibrium point where a

${ }^{21}$ P. W. Bridgman, Proc. Am. Acad. Arts Sci. 74, 1 (1940). 
portion of the sample was already transformed. Davidson and Lee, ${ }^{22}$ also using a hydrostatic system, have measured the sample hysteresis in the conventional manner in which the pressure at the initiation of the transformation on the up and down cycle was measured. They reported hysteresis of from 0.55 to $0.90 \mathrm{kbar}$, which is approximately ten times the region of indifference reported by Bridgman. With the Bi sample surrounded by $\mathrm{NaCl}$, an average hysteresis of $5.3 \mathrm{kbar}$ for a cylindrical wire $0.010 \mathrm{in}$. in diameter and $2.8 \mathrm{kbar}$ for a 0.002 -in. thick flat strip was observed where pressures were measured at the initiation of the transformation. The best explanation of the sample hysteresis measured in this investigation and also by Davidson is found in the nucleation process of the new phase, which would not be of importance in Bridgman's measurement of the "region of indifference" since large amounts of both phases were simultaneously present.

The thermodynamic equilibrium transformation pressure is defined by the equality of the Gibbs free energy for the two phases. If one approaches the equilibrium pressure from phase I, for example, there will exist with increasing probability statistically created nuclei of phase II having some probability distribution with respect to size. When the equilibrium pressure is reached, since the Gibbs free energy in the two phases is equal, the transformation will take place only if the nuclei surface energies are less than or equal to zero so that nuclei growth would result in a lower energy state for the system. In real solids one would expect the nucleus surface energy, which is nothing more than the microscopic strain energy of a nucleus of phase II in a matrix of phase I, to be positive with the result that there is no nuclei growth; hence, the transformation does not occur. In fact it can be easily shown ${ }^{23}$ that the condition for nuclei grow th is that the pressure must be increased above the equilibrium pressure by an amount $\Delta P_{\mathrm{I}-\mathrm{II}}$ such that $G_{\mathrm{I}}(P)-G_{\mathrm{II}}(P)>2 \alpha V_{0} \sigma / R$, where $G_{\mathrm{I}}(P)$ and $G_{\mathrm{II}}(P)$ are the Gibbs functions per atom at a pressure $P$ for the phases I and II, $\sigma$ is the energy per unit surface, $\alpha$ is a factor of order unity which depends on the geometry of the nuclei, $V_{0}$ is the atomic volume, and $R$ is a characteristic dimension of the nuclei. It should be pointed out that this surface energy will vary with (a) the size and shape of the nuclei, (b) the elastic properties and configuration of the surrounding media, (c) the crystal structure and crystalline orientation of the nucleus and its surrounding media, and (d) particularly the volume change associated with the transformation.

It is now easy to understand Bridgman's small "region of indifference": with half of the sample being in phase I and half in phase II, $R$ is very large and hence a very small $\Delta P_{\mathrm{I}-\mathrm{II}}$ and $\Delta P_{\mathrm{II}-\mathrm{I}}$ will satisfy the growth

${ }_{22}^{22}$ T. E. Davidson and A. P. Lee, Trans. Met. Soc. AIME, 230, 1035 (1964).

${ }^{28} \mathrm{R}$. Smoluchowski, Phase Transformations in Solids (John Wiley \& Sons, Inc., New York, 1951), pp. 149-182. inequality for either direction. However, if one is relying on statistically created nuclei where the nuclei have at least a dimension large enough to define a new phase, a hysteresis of $\Delta P_{\mathrm{I}-\mathrm{II}}+\Delta P_{\mathrm{II}-\mathrm{I}}$ characteristic of the nuclei involved will be observed. It should be pointed out that the size distribution of these statistically created nuclei may depend on the purity and shear strain within the sample, and in some instances the probability of creating a minimum nucleus may increase in a strained system. There exists, in addition to the above phenomena, an effect due to pressure gradients that may be produced in solid pressure-transmitting media as a result of the phase transformation itself. If phase II has a smaller volume than phase I and the system is surrounded by a nonhydrostatic solid pressure-transmitting medium, the net result will be that the pressure relative to the bulk material will decrease at the nuclei by $\delta P_{\text {I-II }}$ on the forward transition and increase by $\delta P_{\text {II-I }}$ on the reverse transition by an amount characteristic of the critical nucleus size. The measured sample hysteresis in this case will be the sum $\Delta P_{\text {I-II }}$ $+\delta P_{\mathrm{I}-\mathrm{II}}+\Delta P_{\mathrm{II}-\mathrm{I}}+\delta P_{\mathrm{II}-\mathrm{I}}$.

One must clearly distinguish between the strain energy associated with the nucleation center, as discussed above, and any gross strains or pressure gradients inherent in the apparatus. It must be remembered that the discussion above relates to the balance between growth and decay of nucleation centers, and that at the initiation of the transformation the size of these nuclei are still very small, containing at most a few hundred atoms. ${ }^{23}$ After initiation, the transformation will continue to progress until the pressure within the calibrant and environment decreases because of volume contraction to the point where the growth criterion is violated and then will cease. In order to cause further progress of the transformation in a solid system, one must increase the ram load continuously to cause the transformation to complete. In a good configuration with $\mathrm{Bi}$ (for example) surrounded by $\mathrm{AgCl}$, over half the resistance transition will usually take place following the initiation. The latter "sluggishness" which exists after stabilization following the initiation is well known to workers in the field, and is caused, we propose, by the pressure gradients mentioned.

The existence of the sizable hysteresis in the Bi I-II transformation when measured in a liquid hydrostatic system as reported by Davidson and Lee indicates that the nucleation centers are statistically formed at points within the bulk region of the sample rather than on the surface since any strain energy for a nucleation center on the surface would be relieved by the hydrostatic liquid pressure. Furthermore, since they reported no essential difference between polycrystalline samples and single-crystal samples, one can infer that the nucleation centers are not associated with grain boundaries but rather with lattice vibrations, dislocations, or other crystal defects within the crystal proper.

One would expect a significant increase in the micro- 
scopic strain energy discussed above and thus a change in the hysteresis accompanying the transformation when one includes the strain in the environmental media due to nonhydrostatic conditions inherent in the apparatus. This nucleation phenomenon also explains the smaller hysteresis measured using a flat, metal calibration strip rather than a cylindrical wire since the more favorable configuration would result in reduced pressure gradients around the sample. Although only in the case of $\mathrm{Bi}$ do we have hydrostatic data to support the above interpretation in hysteresis effects, we assume the same basic phenomena are involved in the other transformations reported herein. The $\mathrm{Yb}$ transition exhibits, in addition, a rather large distinctive metastability which might well be due to other phenomena. Since the primary objective of this work was the calibration of the fixed points, thin-strip calibrant specimens were used for all higher pressure calibration studies in order to reduce the hysteresis effects.

The equilibrium values of the transformations were taken as the average of the increasing and decreasing pressure cycle transitions, since there is no way with the present data to ascertain with any certainty in which direction the nucleation energy might be the largest. There is no reason, however, to expect the overpressure in the up cycle and the underpressure in the down cycle to be equal. A slight indication of differing energies in the Bi I-II and Bi II-I transformations can be obtained by noting that the average transition values obtained for the wire calibrant is lower than for the strip calibrant. If one assumes the ratio of the amount of overpressure in the $\mathrm{Bi}$ I-II transition to the amount of underpressure in the $\mathrm{Bi}$ II-I transition is the same for the above situations, the measured average values can be explained by letting this ratio be less than one. This interpretation would mean the nucleation energy in the $\mathrm{Bi}$ I-II transformation is less than in the reverse Bi II-I transformation.

A second minor point of interest arises due to the small pressure interval between the $\mathrm{Bi}$ I-II and $\mathrm{Bi}$ II-III transformations, which implies the nucleation for the Bi II-III transformation probably takes place in an environment which is still under gross strain resulting from the Bi I-II transformation. Similarly, on the pressure decrease cycle the Bi II-I transformation is affected by the Bi III-II transformation. Since strain energy varies as the square of the strain, this would tend to place the Bi II-III transition average higher than its equilibrium value and the $\mathrm{Bi}$ I-II average lower. A perusal of the literature in which both the Bi I-II and Bi II-III transitions are shown on a calibration curve indicates a consistent and significantly larger pressure interval between these two transitions when measured in a solid medium compared to measurements in a hydrostatic medium, a result consistent with the above reasoning. It should be emphasized that the differences implied here are not measurement errors; rather, the initiation of the transformation actually takes place at pressures different from the equilibrium pressures, and the error is in assigning the equilibrium values to the pressure at the transition point. The implications of this are discussed later.

\section{Higher-Pressure Transformations}

The thermodynamic equilibrium pressure value of $24.8 \pm 0.8 \mathrm{kbar}$ (see Table I) obtained for the Bi I-II transformation is in good agreement with the accepted value as determined by more precise techniques in hydrostatic systems. In the light of the discussion in the previous section, the slightly lower value (although within the estimated accuracy) is to be expected. Thus, the Ei I-II transformation results provide good evidence for the feasibility of the calibration technique and also for the accuracy of Decker's theory, at least to $25 \mathrm{kbar}$, and gives one confidence in the method and theory at higher pressures.

Due to the unexpected low value determined for the Ba I-II transition in early experiments, an extensive program was initiated to eliminate possible systematic errors. The transition was measured three or more times with each of two different sized anvils. The different sized anvils required drastically different load pressures, which one might expect to give systematic errors due to warping of the press and associated x-ray geometry. Two different orientations of the $x$-ray system relative to the pressure system were used, and both resistance and volume change associated with the transformation were monitored. A comparison of the measured values under any of these differing conditions showed no variation greater than the estimated error described above. As indicated in Table I the Ba I-II transformation was measured at $54.6 \pm 0.9 \mathrm{kbar}$ and the $\mathrm{Ba}$ II-I at $52.0 \pm 1.5$ kbar. A hysteresis of more than 2 kbar was observed in each measurement with the indicated error being largely a result of experiment-to-experiment variation. Sample hysteresis was also implied by $x$-ray measurements of $\mathrm{Ba}$ compressions in which the compressions at the $\mathrm{Ba} \mathrm{II}-\mathrm{I}$ were smaller than at the $\mathrm{Ba}$ I-II transformation. The resistance of $\mathrm{Ba}$ has been measured to $105 \mathrm{kbar}$ as determined by the $\mathrm{NaCl}$ scale, but the high $\mathrm{Ba}$ transition reported by Balchan and Drickamer ${ }^{9}$ to occur at 144 kbar has not been reached.

The Bi III-V transition was observed to take place at $75.7 \pm 1.3 \mathrm{kbar}$ in three separate experiments, and the reverse transition $\mathrm{Bi}$ V-III was observed at $71.9 \pm 1.3$ $\mathrm{kbar}$ in three determinations. Due to the consistency of these experiments it was felt that no further data were necessary. Again a sizable sample hysteresis was evident. One experimental determination was also made of the transition pressure in thallium, ytterbium, and tin to see if there was general agreement or if serious discrepancies would arise. The data on these three transitions are not intended to represent precise calibration data, but are given rather to indicate the consistency of the data on the $\mathrm{Ba} \mathrm{I}-\mathrm{II}$ and $\mathrm{Bi}$ III-V transformations. Values of $35.4 \pm 2.1 \mathrm{kbar}$ and $33.8 \pm 2.1$ 
kbar were obtained for the TI II-III and the Tl III-II respectively. The decreasing pressure value is rather poorly defined due to a poor-quality $x$-ray diffraction pattern of $\mathrm{NaCl}$ in that particular measurement. The data indicate a rather small hysteresis, however, which is consistent with the small associated volume change. The $\mathrm{Yb}$ I-II transition was carefully measured at the value of $38.2 \pm 1.5 \mathrm{kbar}$ and the reverse $\mathrm{Yb}$ II-I transition at $14.7 \pm 1.1 \mathrm{kbar}$. No equilibrium value is recorded for this transformation because of the pronounced metastability. The Sn I-II transformation ${ }^{24}$ was detected by the structure change in the tin itself, and no resistance measurement was made. Tin powder was intimately mixed with $\mathrm{NaCl}$, and both $\mathrm{x}$-ray patterns were observed simultaneously. A value of $92 \pm 3 \mathrm{kbar}$ was obtained for the Sn I-II transformation, but the reverse Sn II-I transformation was not measured. Thus, no hysteresis value was determined. This is the transformation previously reported at 113 to $115 \mathrm{kbar}$ by Stager, Balchan, and Drickamer ${ }^{25}$ by resistance measurements.

\section{DISCUSSION}

A comparison of the measured transition pressures reported in this work with the accepted scale from Bridgman's volume scale as corrected by Kennedy and LaMori is given in Table I. The differences are surprisingly large even at the Ba transformation, although at lower pressures agreement is much better. The discrepancy between the two scales is shown in graphical form in Fig. 3 to illustrate the smooth departure of the two scales at the higher pressures. In reality the complete volume scale above $40 \mathrm{kbar}$ is based on the $\mathrm{Ba}$ and upper $\mathrm{Bi}$ transformations since the $\mathrm{Sn}$ transformation was tied to $\mathrm{Ba}$ and $\mathrm{Bi}$ by Stager, Balchan, and Drickamer.

In the present work as well as in Bridgman's original volume work measurements were made on both the increasing and decreasing pressure cycles, and an equilibrium value estimated for the transformation. Thus, the curve shown in Fig. 3 corresponds only to equilibrium values. For this reason, the $\mathrm{Yb}$ point is not used. The Sn point is utilized, however, since the measurement by Stager, Balchan, and Drickamer and the measurement reported here were both increasing pressure measurements. Overpressuring effects would thus tend to compensate.

The discrepancy $\left(P_{B}-P_{\mathrm{NaCl}}\right)$ is plotted against Bridgman's pressures in Fig. 3 for convenience in using the curve as a correction curve for data previously reported relative to the volume scale. Curiously, a plot of $\log \left(P_{B}-P_{\mathrm{NaCl}}\right)$ vs $\log \left(P_{\mathrm{NaCl}}\right)$ yields a straight line, implying a power-law relationship. Whether this is coincidental or has significance is not obvious, and we can offer no explanation.

\footnotetext{
${ }^{24}$ J. D. Barnett, V. E. Bean, and H. T. Hall, J. Appl. Phys. 37,875 (1966).

${ }^{25}$ R. A. Stager, A. S. Balchan, and H. G. Drickamer, J. Chem. Phys. 37, 1154 (1962).
}

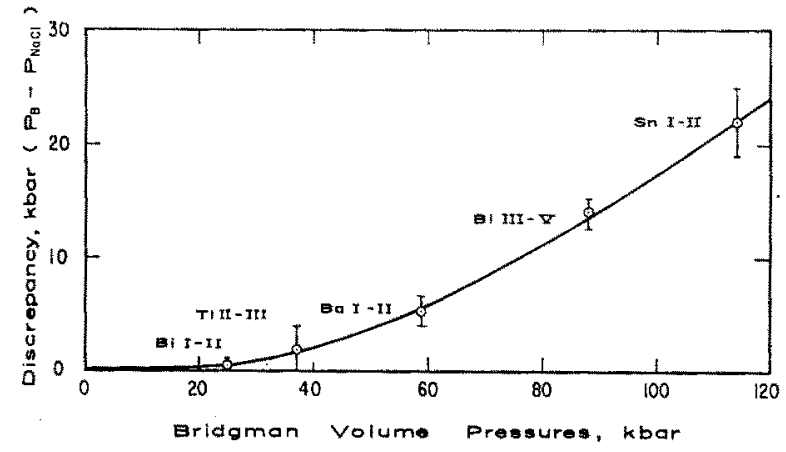

Fig. 3. Differences between $\mathrm{NaCl}$ pressure scale and Bridgman's volume scale. The volume scale used here includes Sn at $114 \mathrm{kbar}$, which was not measured by Bridgman but was determined using apparatus calibration curves based on Bridgman's data.

Since generally in solid-media systems one calibrates on the pressure-increase cycle only, a most important result of this work is the elucidation of the effect of nonhydrostatic environment on the transition pressure. Many workers in the field have been assigning equilibrium pressure values to transitions which have actually taken place at a significantly higher pressure depending on the calibrant environment. If an accurate calibration is to be obtained, some knowledge of the environmental effect must be known. This implies that one does not, in general, know accurately the pressure even if a calibrant is included within the pressure chamber and measurements are made near the transition pressure. It is felt that improved pressure data will result if this problem is understood and some allowance made for it. For standard calibration of solid-media systems we propose the use of flat strip configuration and the use of the transition pressures given in Table I for the pressure increasing cycle rather than the equilibrium pressure value.

Several possible systematic errors which would give rise to the divergence of the higher pressure scales shown in Fig. 3 come to mind.

(1) A systematic error exists in our x-ray geometry at the high load. As mentioned above the use of both small and large tetrahedra sample chambers should have demonstrated the existence of such an error. Furthermore, the sign of the discrepancy observed would indicate that our measured compressions are too small, whereas compression data on $\mathrm{Ba}^{5}$ and $\mathrm{Cs}^{18}$ measured with our apparatus have yielded compressions slightly larger than those reported by Bridgman. In fact, in two experiments simultaneous compression values of $\mathrm{Ba}$ and $\mathrm{NaCl}$ were measured.

(2) The theory used to determine pressures from compressibility data could be in error at the higher pressures. Decker claims an expected pressure accuracy of $2 \%-3 \%$. His estimate is based on agreement with shock data at very high pressures where such data are generally thought to be more accurate, Bridgman's own $\mathrm{NaCl}$ volume data, and the lack of variation due to 
changing parameters in the theory. Further confirmation of Decker's theory is found by comparing the theory with recent data by Perez-Albuerne and 1) rickamer ${ }^{26}$ in which the theory fits the data better than the empirical equation used by the authors. Decker's theory also gives a better fit to the shock data of Christian, ${ }^{27}$ exhibited for comparison by PerezAlbuerne and Drickamer. Since the theory agrees with Bridgman's $\mathrm{NaCl}$ compression values, the present work demonstrates that Bridgman's volume pressures are not consistent but depend upon sample material. One must thus choose to calibrate on Bridgman's $\mathrm{NaCl}$ data or his $\mathrm{Ba}$ and $\mathrm{Bi}$ data where the discrepancies are approximately as shown in Fig. 3. In the absence of a good reason to select one set of Bridgman's data and reject the other, we feel it only consistent to base a calibration on a theoretical curve and thus be completely independent of Bridgman's data. The correlation of Decker's theory with the Bi I-II transition and Christian's shock data does however indicate Bridgman's $\mathrm{NaCl}$ compressibility data is more reliable than his corresponding $\mathrm{Ba}$ and $\mathrm{Bi}$ data.

(3) The third possible explanation of the divergence illustrated in Fig. 3 is that in Bridgman's system an unknown and differing percentage of the load at higher pressures was taken up with friction in measurement of each different sample material. The existence of errors in the correction made to Bridgman's raw data is not at all unlikely. Bridgman himself did not hesitate to correct previous data in light of new measurements in order to obtain consistency. We propose this is necessary at this time. It should be noted that Bridgman never intended these volume measurements to be used for calibration points when the measurements were made. Even a casual reading of his original paper indicates that this was the case. If he had so intended, he would have expended much more effort as he did for the Bi I-II transformation.

Recent results of other workers have also given indications of inconsistencies in the high-pressure range. Klement, Jayaraman, and Kennedy ${ }^{19}$ in a phase diagram of $\mathrm{Bi}$ to $70 \mathrm{kbar}$ found indications that the $\mathrm{Bi}$ III-V phase line extrapolates to room temperature at about 78 to $82 \mathrm{kbar}$. Stark and Jura ${ }^{7}$ and Giardini ${ }^{8}$ have also found indications that $B i$ III $-V$ transition

\footnotetext{
${ }^{26}$ E. A. Perez-Albuerne and H. G. Drickamer, J. Chem. Phys. 43, 1381 (1965).

${ }^{27}$ R. H. Christian, "The Equation of State of the Alkali Halides at High Pressures," Report UCRL-4900, University of California, Lawrence Radiation Laboratory, 1957.
}

takes place at pressures much lower than $88 \mathrm{kbar}$. Since these determinations were themselves based upon the $\mathrm{Ba}$ point at $59 \mathrm{kbar}$, a lowering of this point will reduce the $\mathrm{Bi}$ point even further.

\section{CONCLUSIONS}

Due to the inconsistencies in Bridgman's volume data above $40 \mathrm{kbar}$, we conclude that it is not meaningful to utilize any of his data as calibration points since they were not so intended. Because of the inherent pressure limitations of the piston-cylinder type apparatus and the excessive correction terms involved at the higher pressures, we conclude that a pressure scale must be defined in some manner independent of a given apparatus. We propose an $\mathrm{NaCl}$ pressure scale based upon the linear compression of $\mathrm{NaCl}$ in which pressure is defined by a theoretically predicted equation of state, and we accept Decker's equation as the best available equation evaluated in the pressure region of interest. We propose that where possible, other transitions be related to the compression of $\mathrm{NaCl}$ and that the compression values themselves be reported in the literature, thus tying to a substance rather than a particular theory. We report our determinations of the Ba I-II and $\mathrm{Bi}$ III-V equilibrium transformation pressures at $53.3 \mathrm{kbar}$ and $73.8 \mathrm{kbar}$ as the initial secondary calibration points on such a scale to be improved upon as improvement in technique arises. Data given for $\mathrm{Tl}$, $\mathrm{Yb}$, and $\mathrm{Sn}$ are not so intended. In light of the increasing divergence of the previously accepted scale at higher pressures, we propose an examination of pressure calibration points above $100 \mathrm{kbar}$ along the lines followed here. We further conclude that transition pressures as typically measured in solid-media apparatus are not equilibrium pressures and that measurable and significant errors result if they are thus assumed. The existence of hysteresis effects in the sample itself due to its environment as measured by the initiation of the transformation in the increasing and decreasing pressure cycles has been demonstrated for all transformations studied.

\section{ACKNOWLEDGMENTS}

We wish to thank Dr. Daniel L. Decker for kindly making available the numerical results of his equation of state for $\mathrm{NaCl}$ used in this work and for his interest and encouragement. We also thank Vern Bean and Roy Bennion for assistance in the experimentation. 